\title{
Anchor Movement Strategy for Conjecture Geometry Based Localization Scheme in Wireless Sensor Network
}

\author{
Niraj B Kapase, Santosh Salgar, Mahesh K Patil, Prashant P Zirmite
}

Electronics Department, DKTE's Textile and Engineering Institute Ichalakranji, India

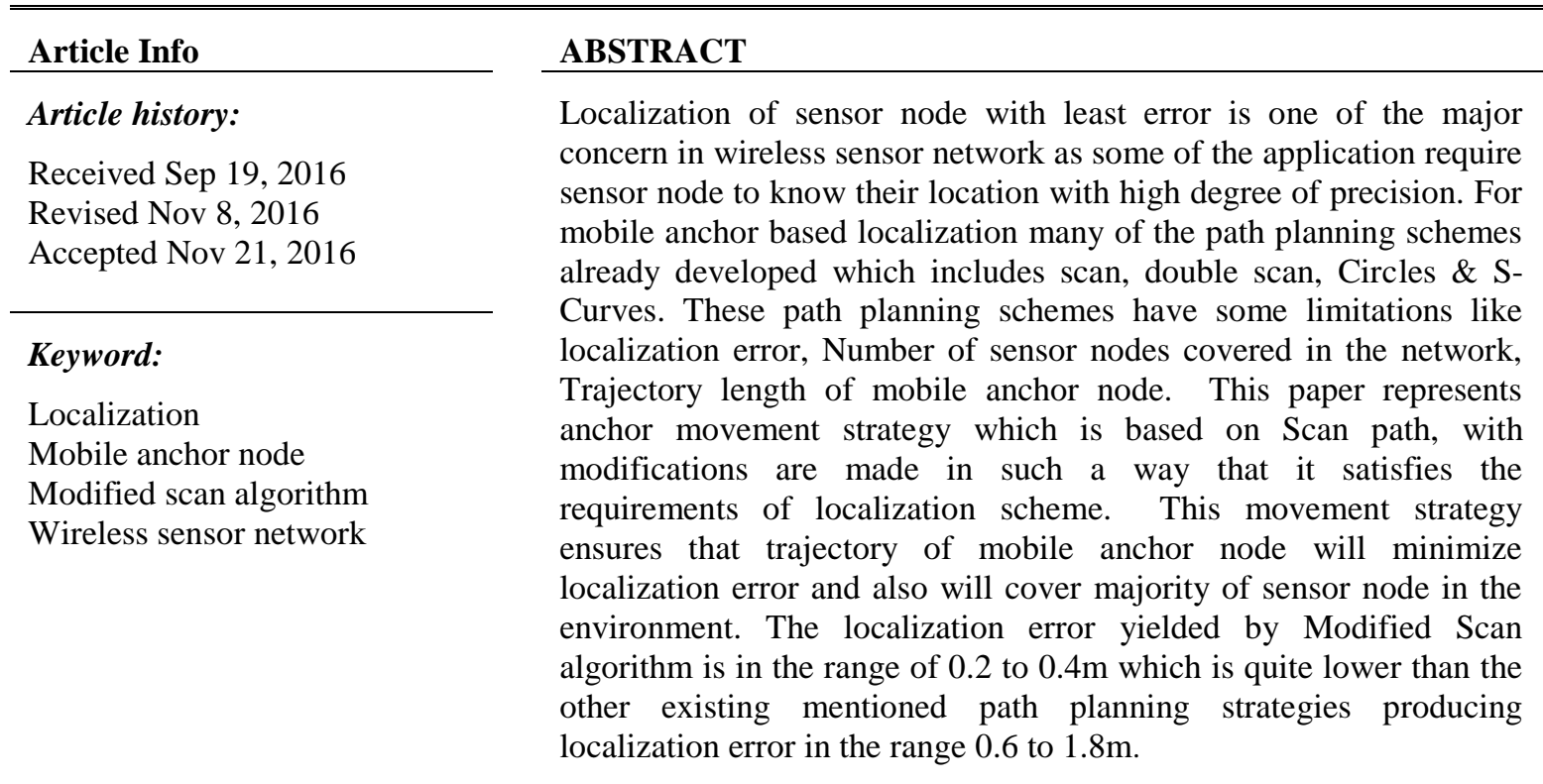

Corresponding Author:

Niraj B Kapase,

Electronics Department,

DKTE's Textile and Engineering Institute Ichalakranji, India

Email: nirajbkapase@dktes.com

\section{INTRODUCTION}

The TA WIRELESS Sensor Network (WSN) consist of hundreds or thousands of sensor nodes and a small number of data collection devices [1]. The sensor nodes are designed to carry out sensing applications including environmental monitoring, military surveillance, fire detection, animal tracking, and so on. The sensor nodes gather the information of interest locally and then forward the sensed information over a wireless medium to a remote data collection device (sink), where it is fused and analyzed in order to determine the global status of the sensed area.

In some WSN applications, the sensor nodes are required to know their locations with a high degree of precision, such as tracking of goods, forest fire detection, and etc. Localization feature work is carried out at network layer. Many localization methods have been proposed for WSNs. Here we are going to deal with mobile anchor based localization scheme which belongs to Range free localization category. In this scheme the GPS-enabled mobile anchor node navigate in sensing field and helps other sensor node tofind their locations. Again different localization algorithms are there under mobile anchor based localization. Several anchor movement strategies have been proposed for all these algorithm. As the anchor movement strategy is developedconsidering specific localization algorithm, their compatibility with other algorithm is not 
guaranteed. In specific we are going to deal with mobile anchor based localization scheme which is based on conjecture geometry. So straight away wecan't use existing path planning strategies such as SCAN, DOUBLE SCAN, HILBERT, S- CURVES which are already developed for other localization scheme as their compatibility with conjecture geometry based localization is not assured.

SCAN cannot guarantee that the length of each chord exceeds a certain threshold. DOUBLE SCAN causes energy and beacon overhead.HILBERTcannot guarantee that every sensor node will obtain the three or more beacon points required to construct two chords of the communication circle. In case of CIRCLE, the path length is extended, and the energy consumption is increased. In S-CURVES, the trajectory of the mobile anchor cannot guarantee that each sensor node can construct two valid chords.

Accordingly this paper presents anchor movement strategy which is compatible with Mobile anchor based localization which isbased on conjecture geometry [2]. The proposed strategy is modified form of existing Scan algorithm which was there for some other localization scheme. The modifications are made considering the methodology of localization.

\section{LOCALIZATION SCHEME}

In the localization scheme [3], a single mobile anchor node moves randomly through the sensing field broadcasting periodic beacon messages containing its current coordinates. Fig. 1 shows the localization scheme based on perpendicular bisector of chord.

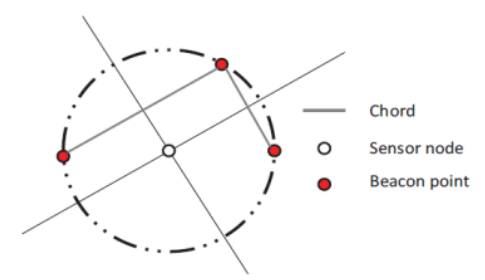

Figure 1. Beacon Points and Chord Construction

The locations of the individual sensor nodes are determined by exploiting the fact that the perpendicular bisector of a chord of a circle passes through the center of the circle. It is assumed that the communication range over which a sensor node can detect broadcasts from the mobile anchor node is bounded by a circle and the sensor node is located at the center of this circle. As the anchor node moves through the sensing field, it broadcasts its coordinates periodically, and each sensor node chooses appropriate locations of the anchor node (called beacon points) to form chords of its communication circle. Once three beacon points (i.e. two chords) have been constructed, the sensor node determines it location by calculating the intersection point of the two perpendicular bisectors of the chords. This method provides a computationally straightforward means of determining the sensor locations.

However, the accuracy of the localization results is dependent on the length of the chords. In realistic environments, the selected beacon points may not be exact on the communication circle. Based on the authors' observation, when the length of the chord is too short, the probability of unsuccessful localization will increase rapidly. Thus, the authors suggested that the length of each chord should exceed a certain threshold in order to minimize the localization error. However, in these scheme the mobile anchor moves randomly through the sensing field (i.e., in accordance with the Random Waypoint model), and thus it is possible that some of the sensor nodes cannot be localized. Therefore, the modified scan algorithm proposed in this study is specifically designed to both minimize the localization error of the individual sensor nodes and to maximize the number of sensor nodes which can determine their locations.

\section{EXISTING PATH PLANNING STRATEGIES}

In this section, it is explained the results of research and at the same time is given the comprehensive discussion. Results can be presented in figures, graphs, tables and others that make the reader understand easily [2], [5]. The discussion can be made in several sub-chapters. 


\subsection{Scan}

In SCAN, the mobile anchor node travels along a single dimension (e.g. the $\mathrm{x}$-axis or $\mathrm{y}$-axis direction), and the distance between two neighboring segments of the node trajectory defines the resolution of the trajectory [4]. SCAN is simple and provides uniform coverage to the entire network. However, the collinearity of the beacons degrades the accuracy of the localization results. SCAN cannot guarantee that the length of each chord exceeds a certain threshold.

\subsection{Double Scan}

In DOUBLE SCAN, the collinearity problem is resolved by driving the anchor in both the $\mathrm{x}$ - and the $y$-directions [4]. However, whilst this strategy improves the localization performance of the sensor nodes, the path length is doubled compared to that of SCAN, and thus the energy overhead increases accordingly. DOUBLE SCAN increases the beacon overhead due to the generation of redundant beacon points.

\subsection{Hilbert}

In HILBERT, the mobile anchor node is driven along a curved trajectory such that the sensor nodes can construct non-collinear beacon points and the total path length is reduced [4]. The results presented showed that through an appropriate setting of the curved trajectory parameters, a significant reduction in the localization error could be obtained compared to the case in which the anchor node simply moved randomly through the sensing field. HILBERT cannot guarantee that every sensor node will obtain the three or more beacon points required to construct two chords of the communication circle.

\subsection{Circle}

In CIRCLES, the mobile anchor follows a sequence of concentric circular trajectories centered at the center point of the deployment area [5]. CIRCLES can only guarantee that the four corners of the sensing field are covered by expanding the diameter of the concentric circles. As a result, the path length is extended, and the energy consumption is increased.

\subsection{S-Curves}

In S-CURVES, the anchor follows an S-shaped curve rather than a simple straight line as in the SCAN method [5]. The results showed that given a trajectory resolution much larger than the radio range, both CIRCLE and S-CURVE schemes cope effectively with the collinearity problem and provide a significantly better localization accuracy and coverage than previous solutions.In S-CURVES, the trajectory of the mobile anchor cannot guarantee that each sensor node can construct two valid chords.

\section{MODIFIED SCAN ALGORITHM}

In order to get at least three beacon points on the communication circle of sensor node to form two chords it is necessary that anchor node must through circle area at least two times.

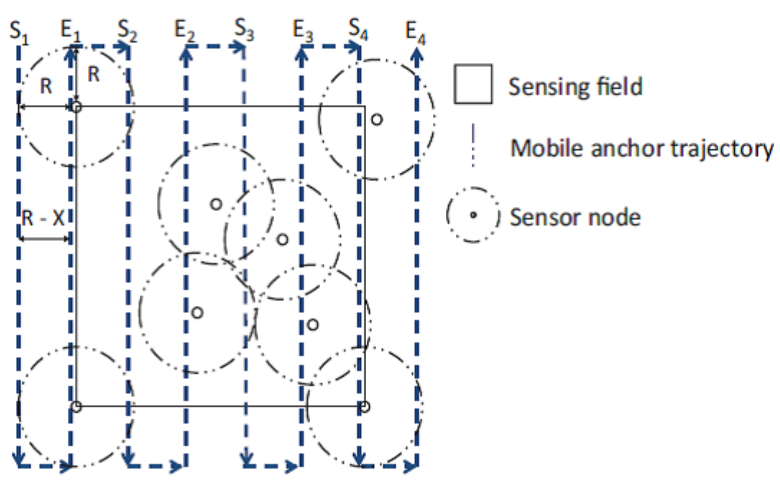

Figure 2. Modified Scan Algorithm

In the modified Scan algorithm proposed in this study, the distance between two successive vertical segments of the anchor trajectory (i.e. the resolution of the anchor trajectory) is specified as $\mathrm{R}-\mathrm{X}$, where $\mathrm{R}$ is the communication radius of the mobile anchor node and $X$ is set in the range $0<X \leq R / 3$. This is because if 
$\mathrm{X}$ is bigger than $\mathrm{R} / 3, \mathrm{R}-\mathrm{X}$ will be smaller than $2 \mathrm{R} / 3$. Hence, the distance between four successive vertical segments is less than the diameter of the communication circle (i.e. $2 \mathrm{R}$ ). As a result, the mobile anchor node will pass through the circle more than three times. In other words, increasing the value of $\mathrm{X}$ may incur redundant beacon points. Conversely, decreasing the value of X may cause the chord length to fall below the minimum threshold value. Thus, in practice, a careful choice of $\mathrm{X}$ is required. To determine the positions of the sensor nodes close to the boundary of the sensing field, the dimensions of the field are virtually extended by a distance of $\mathrm{R}$ on each side, as shown in Fig.2. By extending the sensing field, and choosing an appropriate value of $\mathrm{X}$, the proposed path planning scheme ensures that the mobile anchor node passes through the circle of each sensor node either two or three times.

As shown in Figure 2, the total path length $\mathrm{D}$ is given as

$D=(L+2 R) *\left(\left\lceil\frac{L+2 R}{R-X}\right\rceil+1\right)+(R-X) *\left(\left\lceil\frac{L+2 R}{R-X}\right\rceil\right)$

As the equation (1) indicates, the total path length consists of two components i.e. Vertical path and Horizontal path. The vertical path comprises $\left\lceil\frac{L+2 R}{R-X}\right\rceil+1$ segments of length $(L+2 R)$ and horizontal path comprises $\left\lceil\frac{L+2 R}{R-X}\right\rceil$ segments of length $(R-X)$.

This modified scan algorithms for mobile anchor based localization guarantees following conditions

I. The chord length exceeds $2 \mathrm{R} / 3$

II. All sensor nodes can determine their location

\section{OBSTACLE RESISTANCE STRATEGY}

In a realistic environment, obstacles may appear in the sensing field and thus obstruct the radio connectivity between the anchor node and the sensor nodes. The obstacle-resistant trajectory of the anchor node is introduced in Fig. 3. hen the anchor node moving along the proposed trajectory discovers an obstacle,. After detouring, the anchor node returns to the original proposed trajectory. However, the obstacleresistant trajectory may cause that sensor nodes obtain beacon points which are not on the circle. The localization performance will be degraded due to incorrect beacon points.

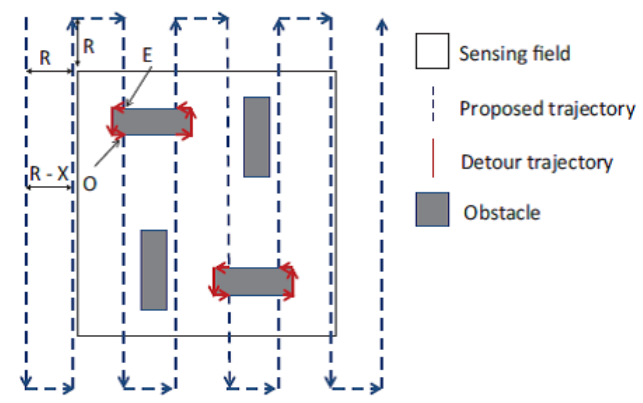

Figure 3.Obstacle Resistance Strategy

\section{PERFORMANE EVALUATION}

The performance of the modified scan algorithm is evaluated by performing a series of simulations on the ns-2 network simulator [6]. The simulation carried out for varying value of X.

1) The localization error: the average discrepancy between the estimated sensor node location and the actual sensor node location for all the sensor nodes;

2) The percentage of localized sensor node: the ratio of the number of successfully localized sensor nodes to the total number of sensor nodes;

3) The chord length: the average chord length constructed by the sensor nodes.

Figure 4 illustrates the localization error of the proposed scheme for various values of $\mathrm{X}$ in the range 0.05 to $0.3 \mathrm{R}$. It can be seen that the localization error maintains a constant value of just $0.2 \sim 0.45 \mathrm{~m}$ as the value of $\mathrm{X}$ is increased. The low value of the localization error arises because the modified scan algorithm guarantees that the length of all the chords constructed by the sensor nodes exceeds $2 \mathrm{R} / 3$ provided that the value of $\mathrm{X}$ is less than $\mathrm{R} / 3$. 


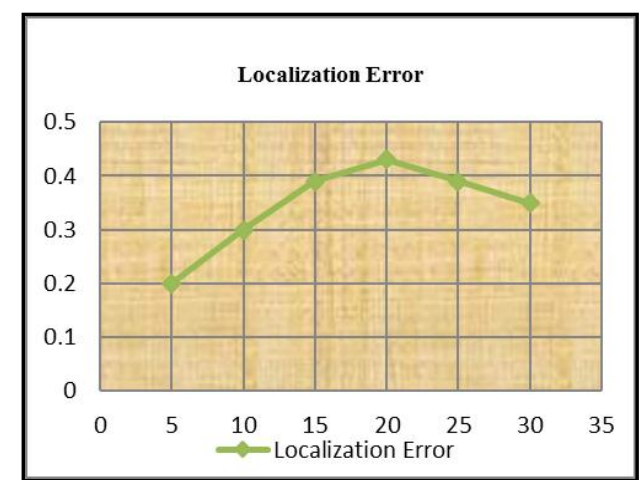

Figure 4. Localization Error (in meter) Vs X

Fig. 5 shows that the average chord length reduces withan increasing value of $\mathrm{X}$. From inspection, the average chord length varies between 25.7 and $28.9 \mathrm{~m}$, and is therefore greater than $2 \mathrm{R} / 3=13.3 \mathrm{~m}$.

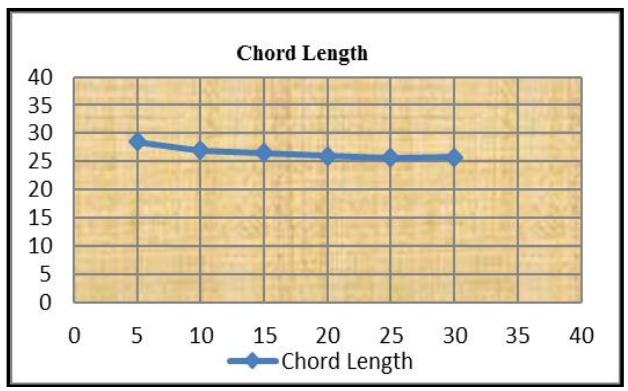

Figure 5. Chord length (in meter) Vs X

Fig. 6 compares the average localization errors of the six path planning schemes in each of the fifty simulation runs. It can be seen that when the presented mobile anchor based localization is implemented using modified scan algorithm, the localization error has a constant value of approximately $0.2 \mathrm{~m}$ and is lower than that achieved by any of the other schemes.

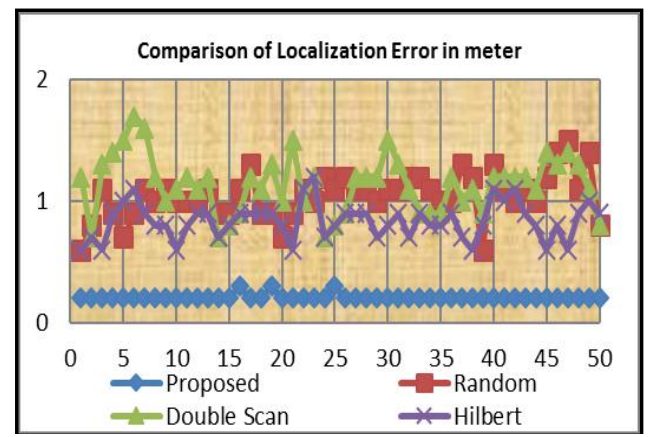

Figure 6. Localization error of all movement strategies

Figure 7 shows the average chord length of the six path planning strategies in each of the fifty simulation runs. The chords constructed by the sensor nodes when using the HILBERT and S-CURVES strategies are significantly longer than those obtained when using the DOUBLE SCAN, CIRCLES and random movement schemes. The chord length obtained when using the modified scan algorithm has a constant value of around $28.5 \mathrm{~m}$ and is comparable to that obtained when using the HILBERT and S-CURVES methods. Of the six schemes, the CIRCLES scheme results in the shortest chord length. 


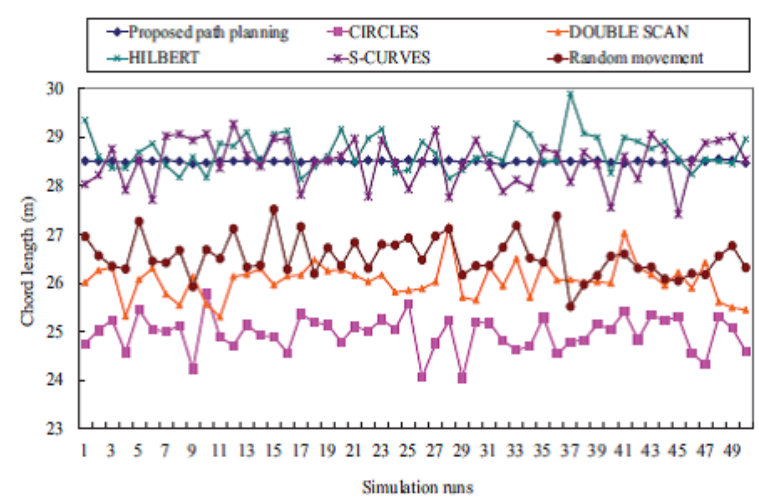

Figure 7. Chord length for all movement strategies

Figure 8 shows the percentage of successfully localized sensor nodes in each simulation run when using the six different path planning strategies. It is observed that the movement strategy proposed in this study enables all of the sensors to determine their locations in every simulation run. It can be seen that DOUBLE SCAN also results in high localization performance (i.e., 98\% 99\%). The remaining movement strategies, i.e., HILBERT, S-CURVES, CIRCLES, and the original random movement strategy, result in average localization percentages of $82 \%, 91 \%, 82 \%$, and $79 \%$, respectively.

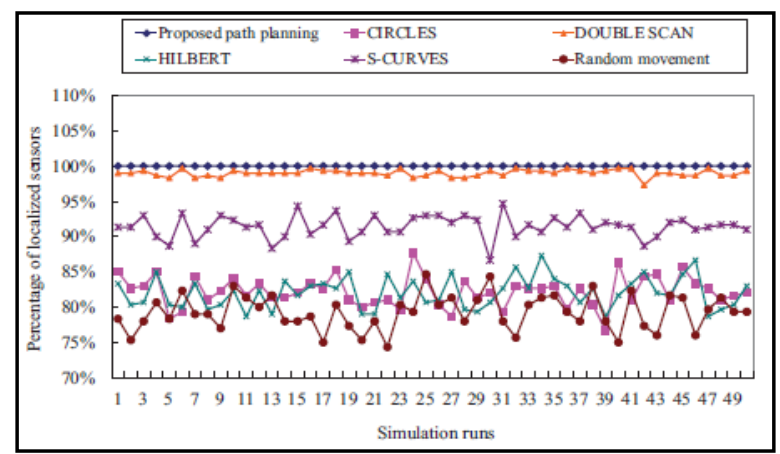

Figure 8. Percentage of localized sensor node

Figure 9 depicts the average localization errors of the obstacle-free and the obstacle environments in each of the fifty simulation runs.Although the virtual beacon point generation is proposed to determine the locations of sensor nodes, the estimated position of the virtual beacon point may not be correctly located on the circle and thus incurs more errors on localization. It can be seen that the localization error of the obstaclefree environment keeps about $0.2 \mathrm{~m}$ and the obstacle environment has a localization error between 0.6 to $0.8 \mathrm{~m}$.

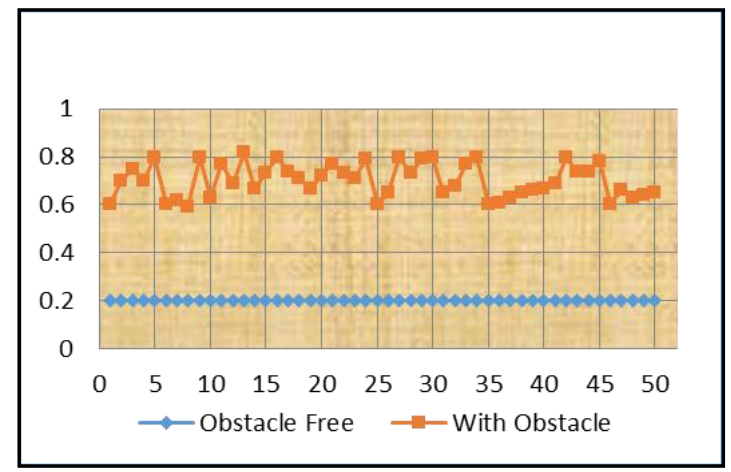

Figure 9.Localization error for Obstacle Free and Obstacle Environment (in meter) 
Figure 10 shows that the average chord length of the obstacle-free and the obstacle environments in each of the fifty simulation runs. The average chord length of the obstacle environment varies between 29.5 and $30.8 \mathrm{~m}$ and is larger than that of the obstacle-free environment

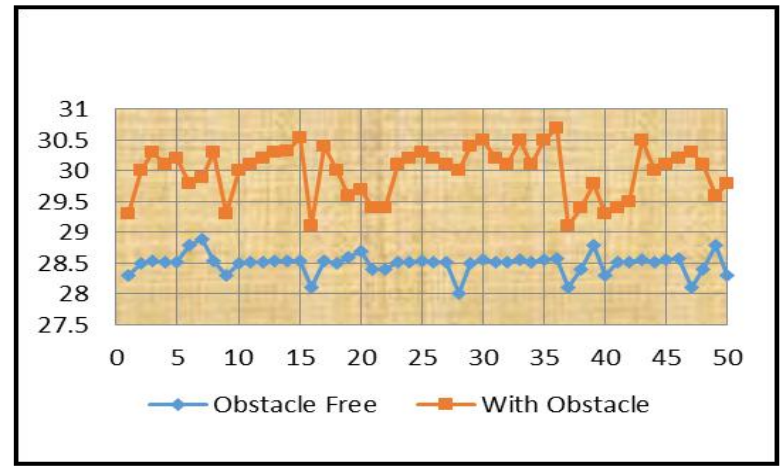

Figure 10. Chord length of obstacle free and obstacle environment (in meter)

\section{CONCLUSION}

In this paper, we have presented anchor movement strategy modified scan algorithm for the mobile anchor node in the localization method based on conjecture geometry. This algorithm ensures that the chords constructed by the individual sensor nodes always have a length greater than $2 \mathrm{R} / 3$. Thus, the short chord problem is is resolved. Besides, the modified movement trajectory and the virtual beacon point generation scheme are presented to tolerate the obstacles in the sensing field. The performance of the modified scan algorithm is compared numerically with that of five existing path planning schemes, namely DOUBLESCAN, CIRCLES, S-CURVES, HILBERT, and the original random movement strategy. Overall, the simulation results have shown that the modified scan algorithm outperforms existing methods in terms of both a smaller localization error and a higher percentage of successfully localized sensor nodes. The localization error yielded by Modified Scan algorithm is in the range of 0.2 to $0.4 \mathrm{~m}$ which is quite lower than the other existing mentioned path planning strategies producing localization error in the range 0.6 to $1.8 \mathrm{~m}$

\section{FUTURE SCOPE}

The improvement in chord length variation for obstacle environment can be achieved with further modification in Beacon point selection mechanism.

\section{REFERENCES}

[1] I. F. Akyildiz, W. Su, Y. Sankarasubramaniam, and E. Cayirci, "A survey on sensor networks," IEEE Commun. Mag., vol. 40, no. 8, pp. 102-114, Auguest 2002.

[2] Chia-Ho Ou, Membr, IEEE, and Wei-Lun He, "Path Planning Algorithm for Mobile Anchor-Based Localization in Wireless Sensor Networks," IEEE Sensors Journal, vol. 13, no. 2, pp. 466-475, February 2013.

[3] C. H. Ou, and H. C. Jiau K. F. Ssu, "Localization with mobile anchor points in wireless sensor networks," IEEE Trans. Veh. Technol, vol. 54, no. 3, pp. 1187-1197, May 2005.

[4] S. M. Das, and Y. C. Hu D. Koutsonikolas, "Path planning of mobile landmarks for localization in wireless sensor networks," Comput. Commun, vol. 30, no. 13, pp. 2577-2592, sept. 2007.

[5] R. Huang and G. V. Zaruba, Static path planning for mobile beacons to localize sensor networks, in Proc. IEEE Int. Conf. Pervas. Comput. Commun. Workshops, Mar. 2007, pp. 323-330.

[6] "The Network Simulator - ns-2," http://www.isi.edu/nsnam/ns/, (2012). 